\title{
Diversity of Groundwater Crustaceans in Wells in Various Geologic Formations of Southern Poland
}

\author{
Agnieszka Pociecha $^{1, *(\mathbb{D}}$, Maciej Karpowicz ${ }^{2} \mathbb{D}$, Tadeusz Namiotko $^{3} \mathbb{D}$, Elżbieta Dumnicka ${ }^{1}(\mathbb{D})$ and \\ Joanna Galas ${ }^{1}$ (D) \\ 1 Institute of Nature Conservation, Polish Academy of Sciences, Adam Mickiewicz 33, 31-120 Kraków, Poland; \\ dumnicka@iop.krakow.pl (E.D.); galas@iop.krakow.pl (J.G.) \\ 2 Department of Hydrobiology, Faculty of Biology, University of Białystok, Ciołkowskiego 1J, 15-245 Białystok, \\ Poland; m.karpowicz@uwb.edu.pl \\ 3 Laboratory of Biosystematics and Ecology of Aquatic Invertebrates, Department of Evolutionary Genetics \\ and Biosystematics, Faculty of Biology, University of Gdansk, Wita Stwosza 59, 80-308 Gdansk, Poland; \\ tadeusz.namiotko@ug.edu.pl \\ * Correspondence: pociecha@iop.krakow.pl
}

Citation: Pociecha, A.; Karpowicz, M.; Namiotko, T.; Dumnicka, E.; Galas, J. Diversity of Groundwater Crustaceans in Wells in Various Geologic Formations of Southern Poland. Water 2021, 13, 2193. https://doi.org/10.3390/ w13162193

Academic Editor: Jun Yang

Received: 18 July 2021

Accepted: 9 August 2021

Published: 11 August 2021

Publisher's Note: MDPI stays neutral with regard to jurisdictional claims in published maps and institutional affiliations.

Copyright: (c) 2021 by the authors. Licensee MDPI, Basel, Switzerland. This article is an open access article distributed under the terms and conditions of the Creative Commons Attribution (CC BY) license (https:// creativecommons.org/licenses/by/ $4.0 /)$.

\begin{abstract}
Data on Crustacea from underground waters accessed through wells are limited in Poland. A recent study was undertaken to determine diversity and factors influencing the crustacean communities inhabiting wells drilled in three bedrocks, Jurassic limestone, Cretaceous marls and flysch. A total of 23 crustacean species and subspecies were recorded belonging to Copepoda, Ostracoda, Amphipoda and Bathynellacea. Only four species of low abundance, however, were stygobionts. Our studies showed that abundance and species number of Copepoda and Ostracoda were affected by bedrock geology (with higher abundances and species richness in wells of Cretaceous marls), and in the case of copepods, also by sampling season. Furthermore, this paper lists all species of Crustacea recorded from inland groundwater habitats of Poland based published over the last 133 years. The most species-rich group was Copepoda with 43 representatives (four stygobites), followed by Ostracoda and Amphipoda with a total of 37 and 12 species, respectively (each with nine stygobites). In addition, two species of Isopoda (one stygobite) and one Bathynellid appear in the checklist. The checklist identifies geographical (and environmental) gaps which require further research.
\end{abstract}

Keywords: copepods; ostracods; subterranean crustacean checklist; ecology

\section{Introduction}

The subterranean aquatic environment represented by cave waters, dug or drilled wells, interstitial waters and hypotelminorheal [1] is the habitat of a range of invertebrates. Among them, crustaceans arprovude the largest number of stygobiontic species [1-3] often accompanied by epigean species.

Wells are a source of drinking water in African countries which is why their fauna has been frequently studied and contains numerous stygobiontic Crustacea [4]. In European countries, studies on crustacean fauna were undertaken in dug wells in former Czechoslovakia [5-7], in boreholes in Germany [8] and in both types in Ireland [9].

In Poland, studies on aquatic subterranean crustaceans started in wells, when Wrześniowski [10] described Niphargus tatrensis and Jaworowski [11] published the results of his invertebrate investigations in Kraków and Lvov.

More recently, other researchers from Poland studied particular crustacean groups in this habitat: Isopoda [12,13], Copepoda [14-20] and Ostracoda [21-28]. The Amphipoda of wells (beside Wrześniowski [10]) were studied by Haeckel [29], Micherdziński [30] and Skalski [31,32] who also summarised the state of knowledge concerning the distribution of this group in Poland [33-35]. Studies on more than one crustacean group in wells are rare $[36,37]$. The mentioned studies were restricted to one region of Poland and to single 
wells, and the knowledge on crustaceans inhabiting subterranean waters in Polish wells therefore remained poor (except for the genus Niphargus—see Dumnicka and Galas [38]).

In rural areas situated in southern Poland, there are numerous old dug wells, mainly unused presently. Access to them gives the possibility to study subterranean aquatic invertebrates in regions of different geological character, even in areas without caves. During the macroinvertebrate studies from 26 wells in southern Poland, only one crustacean species Niphargus tatrensis was determined [3].

The biodiversity of groundwater in Poland remains poorly known compared to that of freshwater surface habitats, so the aim of our studies, conducted in 2010-2016 was to fill this gap in information on the diversity of crustaceans inhabiting groundwater. We also tested the hypothesis that geological bedrock type (Jurassic limestone, Carpathian flysch and Cretaceous marls) in which wells were dug as well as the sampling season (month) influence abundance, diversity and composition of crustacean assemblages.

\section{Materials and Methods}

\subsection{Study Area}

Our study included 33 wells distributed in three geologically different regions viz. (1) the Jurassic limestone area of the Kraków-Częstochowa Upland (14 wells in Szklary and Witkowice villages), (2) the Cretaceous marls of the Miechów Upland (7 wells in Prandocin village) and (3) the flysch areas of the Pogórze Wiśnickie foothills (7 wells in Kawec) and the Beskid Mały mountains (5 wells in Jaszczurowa village) (Figure 1, Table 1).

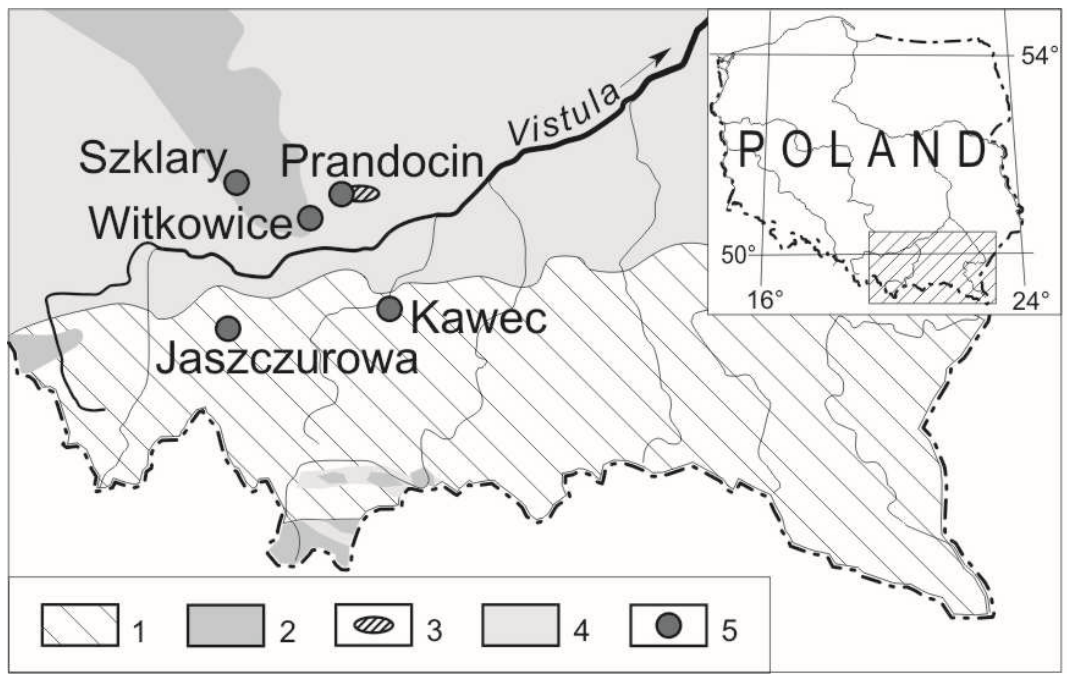

Figure 1. The study area with locations of villages where the studied wells are situated.

Bedrock includes flysch rocks of the Carpathians (1), Jurassic limestone sedimentary rocks (2), Cretaceous marls (3) and other sedimentary rocks of various ages (4). The dots (5) indicate villages with the sampled wells.

Table 1. Localization of villages with data on the sampled wells.

\begin{tabular}{cccccc}
\hline Localization & Coordinates & Geology Type & $\begin{array}{c}\text { No of Studied } \\
\text { Wells }\end{array}$ & Dates of Samplings & Depth (m) \\
\hline Szklary (Sz) & $50^{\circ} 10^{\prime} \mathrm{N} ; 19^{\circ} 42^{\prime} \mathrm{E}$ & Jurassic limestone & 7 & June, August, October 2010 & $1.5-20$ \\
Witkowice (Wi) & $50^{\circ} 10^{\prime} \mathrm{N} ; 19^{\circ} 94^{\prime} \mathrm{E}$ & Jurassic limestone & 7 & May, July, October 2012 & $4.5-20$ \\
Jaszczurowa (Ja) & $49^{\circ} 47^{\prime} \mathrm{N} ; 19^{\circ} 30^{\prime} \mathrm{E}$ & Carpathian flysch & 5 & June, August, November 2012 & $1-4$ \\
Kawec (Ka) & $49^{\circ} 84^{\prime} \mathrm{N} ; 20^{\circ} 22^{\prime} \mathrm{E}$ & Carpathian flysch & 7 & May, July, October 2013 & $1.9-12.5$ \\
Prandocin (Pr) & $50^{\circ} 15^{\prime} \mathrm{N} ; 20^{\circ} 06^{\prime} \mathrm{E}$ & Cretaceous marls & 7 & May, August 2016 & $6-14$ \\
\hline
\end{tabular}




\subsection{Sampling and Measurements of Water Properties}

Samples of invertebrate fauna and water for chemical analyses were collected from $5-7$ wells in each of the five particular regions (33 wells in total, see Figure 1) in 20102016 (Table 1). In each locality, wells were sampled seasonally three times a year (from May to November), except for wells in the Prandocin village where samples were taken on two occasions (Table 1). Thus, the sampling effort included a total 92 samples. The depth of the studied wells differed in particular regions ranging from 1.5 to $20 \mathrm{~m}$. Only in the Jaszczurowa village were all studied wells relatively shallow, i.e., not exceeding $4 \mathrm{~m}$ (Table 1). Most of the studied wells were located in agricultural areas in small farms where non-intensive farming methods were used. Except for wells in the Witkowice village, they were all situated in gardens. The water in the studied wells is not drinking water, farmers use the water from the wells for garden watering and irrigation, which causes fluctuations in the water level, and some of them are not used at all.

Methods used for analyses of physical and chemical water feature analyses of the investigated wells have been described in Dumnicka et al. [3]. The results of studies on benthic invertebrates other than Crustacea (Copepoda and Ostracoda) from the same wells have already been published [3], while the data on plankton samples from wells from the area of Jaszczurowa village were presented as a conference poster [39].

Qualitative samples of Copepoda were taken by a plankton net (50 $\mu \mathrm{m}$ mesh size), using vertical hauls from the well bottom. Benthic samples were taken by an Ekman sampler $(20 \times 20 \mathrm{~cm})$ and filtered through $0.3-\mathrm{mm}$ net mesh. All samples were preserved in $4 \%$ formaldehyde, and fauna was determined using selected keys: for Copepoda e.g., [18,40-42] and Ostracoda [23,43-45]. Prior to the identification, ostracods (intact complete specimens with limbs as well as empty carapaces and valves) were rinsed in water, transferred to $96 \%$ ethanol and then analysed following Namiotko et al. [46]. Investigated specimens were identified to the species or the lowest possible taxonomic level (genus).

\subsection{Statistical Analyses}

To evaluate if the sampling effort was sufficient to represent biodiversity of the crustacean assemblages in the studied area, we performed accumulation curves of the observed and estimated species number by the Chao 1 index using PRIMER 7 software [47].

Other statistical analyses were performed with XLSTAT Ecology (Addinsoft). We used two-way unbalanced ANOVA to determine (i) the effect of physical and chemical variables, and (ii) geology with sampling season (month) on the Copepoda and Ostracoda communities (abundance, number of species). We used the same approach to test the effect of the above factors on the dominant species/genus. To find out if the two or more variables, and their interaction, provide the same amount of information we used Type I SS (sum of squares). For pairwise differences between means, we used Tukey's HSD (honestly significantly different) test. The most important differences were presented by box plots with basic descriptive statistics. The samples without Copepoda and Ostracoda were excluded from the statistical analysis.

\section{Results}

\subsection{Physical and Chemical Water Properties of the Studied Wells}

Water of the wells was circumneutral to alkaline, and mean values ranged from 7.0 in the Ka wells to 7.5 in the $\mathrm{Sz}$ wells. The other parameters of water such as conductivity, $\mathrm{Ca}^{2+}, \mathrm{NO}_{3}{ }^{-}, \mathrm{O}_{2}, \mathrm{SO}_{4}{ }^{2-}$ and $\mathrm{Cl}^{-}$in the studied wells differed strongly (Figure 2). The mean value of conductivity in Jurassic limestone varied from 606 to $1006 \mu \mathrm{S} / \mathrm{cm}$, in flysch regions from 286 to $706 \mu \mathrm{S} / \mathrm{cm}$, and in Cretaceous marls $917 \mu \mathrm{S} / \mathrm{cm}$. The mean values of calcium concentrations in Jurassic limestone and Cretaceous marls areas were higher than in flysch areas, though in water in Ka wells this parameter reached high concentration values. The mean oxygen concentration was high in all wells in Pr, whereas in Wi it was mostly relatively low (Figure 2). In the remaining regions, the mean value of this parameter varied from well to well e.g., in Sz from 9.76 in well no 6 to 3.36 in well no 3 whereas in Ja 
from 8.27 in well no 1 to 0.96 in Well No. 4. Similar fluctuations were observed for nitrates, especially in the water of Wi wells (Figure 2), with the lowest values recorded in the flysch area. Chloride concentrations were low and constant in Ja, while most variable in Wi (Figure 2). In Pr and Ja, the content of sulphates was leveled but in Pr it was relatively high while and in Ja, low. In the remaining three villages the values of this parameter varied strongly. Polluted wells influenced by antropogenic factor occurred in several studied regions, but most often in Wi and Pr.

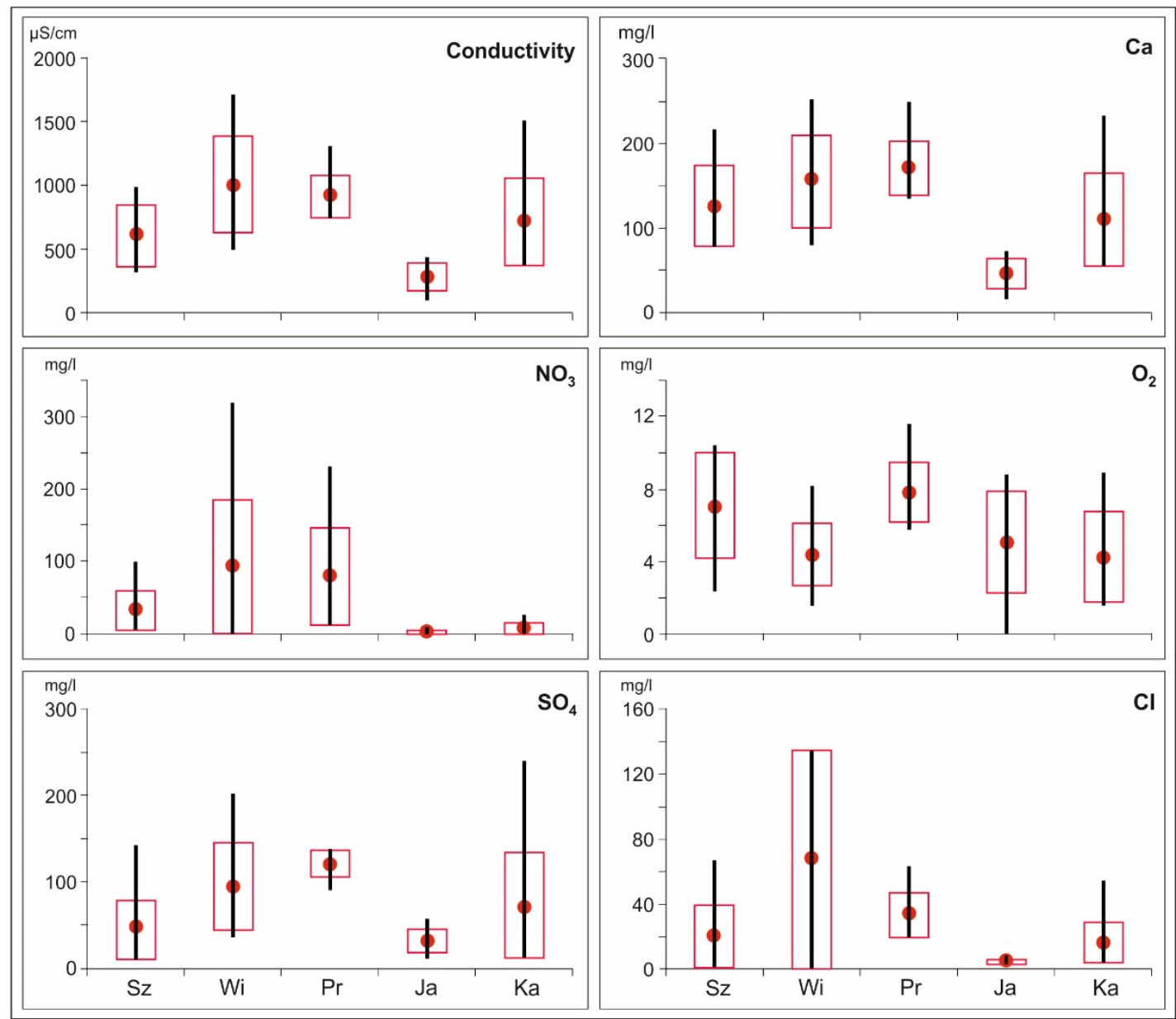

Figure 2. Selected water physical and chemical parameters: mean (in the middle of box), $\pm S D$ (box), max and min-whiskers. Wells in Sz and Wi are in Jurassic limestone bedrock, those in Pr are located in Cretaceous marls, whereas wells in Ja and Ka are in flysch (see Table 1 for village codes and other details).

\subsection{Crustaceans in the Studied Wells}

A total of 23 crustacean taxa of the ranks of species and subspecies were recorded (some were left in open nomenclature). They belonged to Copepoda, Ostracoda, Amphipoda and Bathynellacea. Although the accumulation plot of the observed species number did not reach asymptotic levelling-off (Figure 3), the total observed crustacean species richness was $73.5 \%$ of the species number estimated by the Chao 1 index (mean \pm standard deviation $\mathrm{SD}=32.7 \pm 10.27)$.

In the studied wells, 13 copepod taxa were stated. Cyclopoida were represented by ten taxa of the species group (Table 2). Only three species were most abundant: Diacyclops bisetosus (from 7 to 34 individuals in the wells of Sz and Pr villages), Acanthocyclops vernalis (from 1 to 24 ind.-Ja, Pr), and Megacyclops viridis (from 1 to 15 ind.-Ja) and these species were also the most frequent. Only three Harpacticoida species were determined including stygobiontic species Elaphoidella elaphoides. In four wells, only cyclopoid nauplii and/or 
copepodites was identified, and in one well, only harpacticoid copepodites were presented (Table 2).

Altogether, eight species of Ostracoda were recorded during this survey (Table 2, Cryptocandona sp. is considered to represent juveniles of Cryptocandona matris), of which three (and the mentioned Cryptocandona sp.) remained in open nomenclature due to a poor preservation state and/or juvenile stage preventing certain identification. Cryptocandona matris (including Cryptocandona sp.) and Typhlocypris cf. eremita (both belonging to family Candonidae) can be regarded as stygobiontic species. The latter species and Cavernocypris subterranea were the most common, both with records from five wells, while six other species were found only in one well. The maximum number of species reported in a single well (Ka6) was four (C. matris, Cyclocypris ovum, Cypria ophtalmica and Fabaeformiscandona brevicornis), whereas the most abundant ostracod samples were taken from Pr wells.

Among amphipods, singular specimens of Niphargus tatrensis were found in two wells. Finally, one species of bathynellaceans, Bathynella natans, was recorded in the Prandocin wells (Table 2).

Table 2. Crustacea recorded in the studied wells.

\begin{tabular}{|c|c|c|c|c|c|c|c|}
\hline \multirow{2}{*}{$\begin{array}{c}\text { Crustacean } \\
\text { Groups }\end{array}$} & \multirow[t]{2}{*}{ Order } & \multirow[t]{2}{*}{ Taxa and Life Stages of Invertebrates } & \multicolumn{5}{|c|}{ Wells } \\
\hline & & & Szklary & Jaszczurowa & Kawec & Witkowice & Prandocin \\
\hline \multirow[t]{16}{*}{ Copepoda } & Cyclopoida & nauplii Cyclopoida & + & + & & & + \\
\hline & & copepodids Cyclopoida & + & + & & + & + \\
\hline & & Acanthocyclops kieferi (Chappuis, 1925) & & & & + & \\
\hline & & Acanthocyclops vernalis (Fischer, 1853) & & + & & & + \\
\hline & & Acanthocyclops robustus (Sars G.O., 1863) & & & & + & \\
\hline & & Diacyclops sp. & & & & + & \\
\hline & & Diacyclops bisetosus (Rehberg, 1880) & + & & & & + \\
\hline & & Diacyclops crassicaudis (Sars G.O., 1863) & + & & & & + \\
\hline & & Diacyclops crassicaudis brahycercus (Kiefer, 1927) & & & & & + \\
\hline & & Megacyclops viridis (Jurine, 1820) & & + & & & \\
\hline & & Paracyclops imminutus Kiefer 1929 & & + & & & \\
\hline & & Tropocyclops prasinus (Fischer,1860) & & + & & & \\
\hline & Harpacticoida & copepodids Harpacticoida & & & & & + \\
\hline & & Elaphoidella elaphoides (Chappuis, 1923) & & & & & + \\
\hline & & Elaphoidella cf. elaphoides & & & + & & \\
\hline & & Mesochra sp. (Schmeil, 1894) & & & & & + \\
\hline \multirow[t]{9}{*}{ Ostracoda } & & Cavernocypris subterranea (Wolf, 1920) & + & & & & + \\
\hline & & Cryptocandona matris (Sywula, 1976) & & & + & & \\
\hline & & Cryptocandona sp. & & & + & & \\
\hline & & Cyclocypris ovum (Jurine, 1820) & & & + & & \\
\hline & & Cyclocypris cf. serena (Koch, 1838) & & & & + & \\
\hline & & Cypria ophtalmica (Jurine, 1820) & & & + & & \\
\hline & & Fabaeformicandona brevicornis (Klie, 1925) & & & + & & \\
\hline & & Potamocypris cf. pallida Alm, 1914 & & & & & + \\
\hline & & Typhlocypris cf. eremita (Vejdovsky, 1882) & + & & & & + \\
\hline Bathynellacea & & Bathynella natans Vejdovsky, 1882 & & & & & + \\
\hline Amphipoda & & Niphargus tatrensis Wrześniowski, 1888 & & & + & & + \\
\hline Sum of taxa & & & 4 & 4 & 7 & 4 & 11 \\
\hline
\end{tabular}

+ -presence taxon confirmation.

\subsection{Statistical Analyses}

The physical and chemical variables did not significantly affect the abundance of Copepoda $(\mathrm{F}=0.63 ; p=0.81)$ and Ostracoda $(\mathrm{F}=1.57 ; p=0.22)$, as well as the number of Copepoda $(\mathrm{F}=0.96 ; p=0.54)$ and Ostracoda species $(\mathrm{F}=2.56 ; p=0.06)$. The abundance of dominant Copepoda and Ostracoda species also was not significantly affected by water properties. Only the abundance of the Acanthocyclops species was affected by these variables $(\mathrm{F}=11.3 ; p<0.0001)$. The Type I SS analysis indicated that Acanthocyclops abundance was affected by temperature $(p<0.0001)$, phosphates $(p<0.0001)$, nitrates $(p<0.0001)$, dissolved oxygen $(p=0.003)$, electrical conductivity $(p=0.022)$, and sulphates $(p=0.048)$.

We found that both the geology and the sampling season (month) significantly affected the abundance of Copepoda $(\mathrm{F}=4.15 ; p=0.0003)$ and Ostracoda $(\mathrm{F}=5.54 ; p<0.001)$, number 
of species of Copepoda ( $\mathrm{F}=3.58 ; p=0.001)$ and Ostracoda $(\mathrm{F}=5.72 ; p<0.001)$, as well as the abundance of dominant species (Figures 4 and 5). Only Ostracoda abundance (Figure 4B) and number of species (Figure 4D) were not affected by months. Copepoda highest abundance (Figure 4A) and number of species (Figure 4C) were found in September. The Cretaceous marls and Carpathian flysch had a higher abundance of Copepoda, and a higher number of species than Jurassic limestone (Figure $5 \mathrm{~A}, \mathrm{C}$ ). Ostracoda had the highest abundance and number of species in Cretaceous marls (Figure 5B,D).

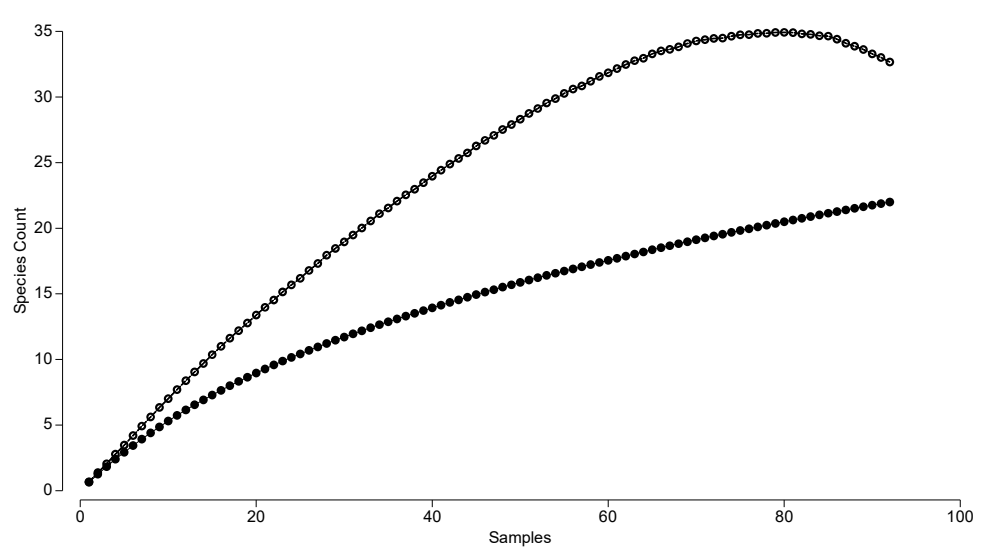

Figure 3. Species accumulation plot based on all 92 studied samples. Closed circles-number of observed species, open circles-number of estimated species by the Chao 1 index.

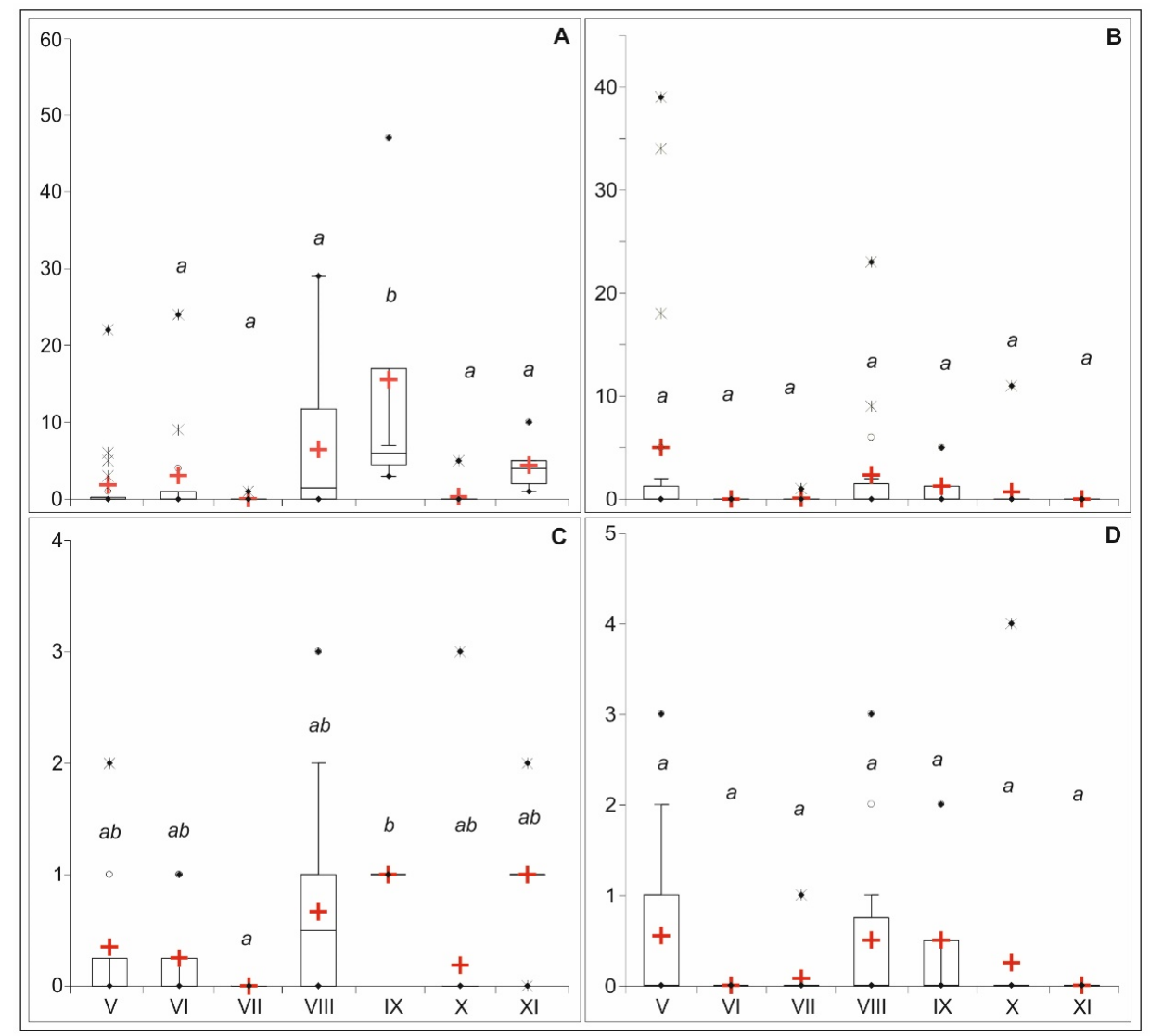

Figure 4. Monthly differences in Copepoda abundance (A), Ostracoda abundance (B), number of Copepoda species (C), number of Ostracoda species (D) in the studied wells. The different letters $(a, b)$ above the box plots denote significantly different values at $p<0.05$ and the same letters denote no statistically significant differences. The limits of the boxes are the first and third quartiles, crosses represent the means, central horizontal bars are the medians, points above or below are outliers, and the whiskers represented min and max. 


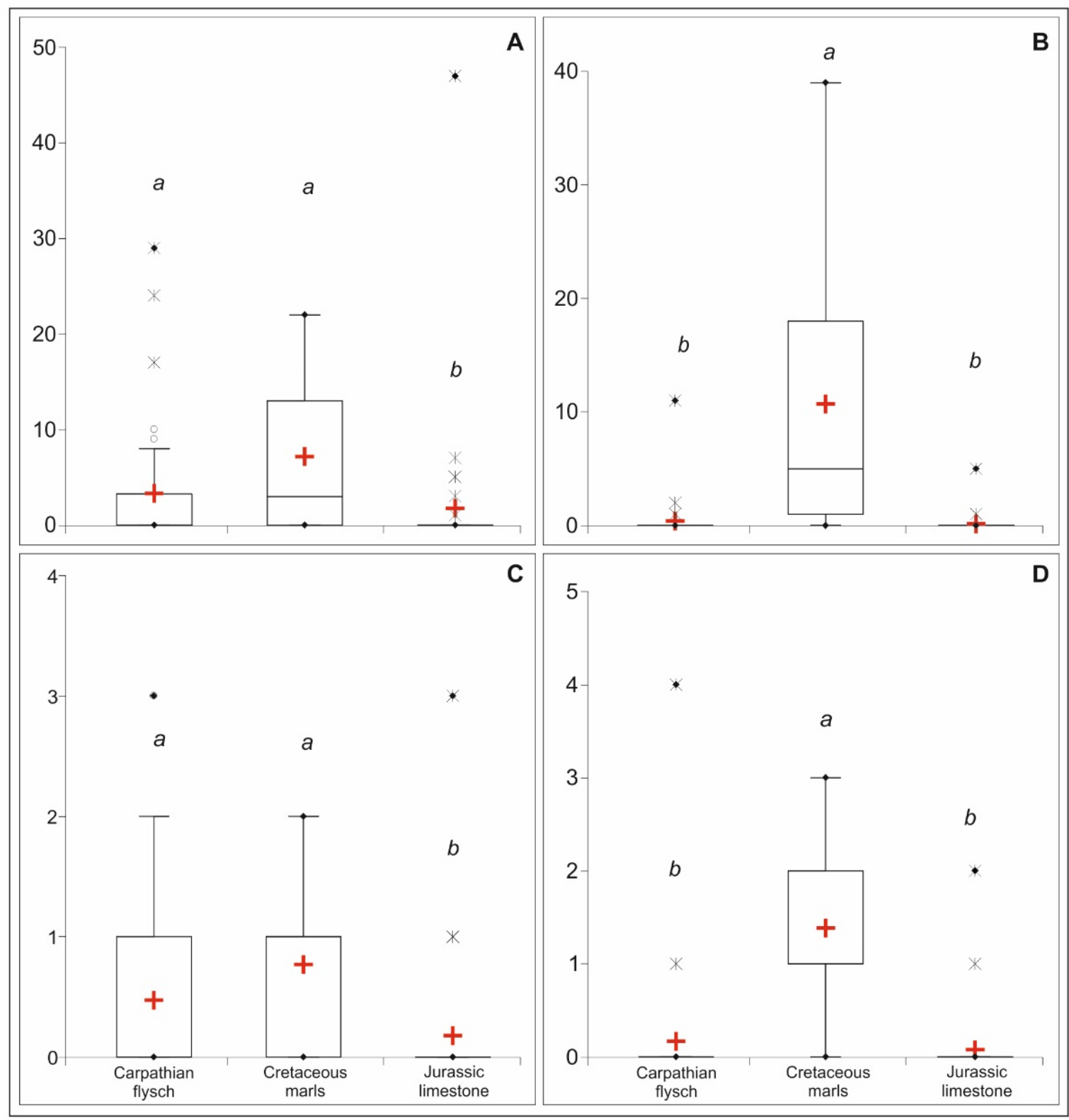

Figure 5. The effect of geology on Copepod abundance (A), Ostracoda abundance (B), number of Copepoda species (C), number of Ostracoda species (D) in the studied wells. The different letters $(a, b)$ above the box plots denote significantly different values at $p<0.05$ and the same letters denote no statistically significant differences. The limits of the boxes are the first and third quartiles, crosses represent the means, central horizontal bars are the medians, points above or below are outliers, and the whiskers represent min and max.

The geology and months also significantly affected the abundance of dominant species/genus. Diacyclops was affected by months $(p=0.001)$ and geology $(p=0.044)$, with the highest abundance in September and Cretaceous marls. Megacyclops viridis was affected by geology $(p=0.031)$ with the highest abundance in Carpathian flysch. We did not find a significant effect of geology and month on the abundance of Acanthocyclops genus. Concerning ostracods, Cavernocypris subterranea and Typhlocypris cf. eremita abundances were significantly affected by the geology $(p<0.0001)$ with the highest abundance of both species in Cretaceous marls. 


\subsection{Crustacea in Subterranean Waters of Poland-State of Current Knowledge}

The literature concerning Crustacea recorded from subterranean waters (including caves, wells and interstitial waters) of Poland is limited. Since Wrześniowski's classic work [10], in which two amphipod species new to science (Niphargus tatrensis and Synurella tenebrarum) were described, 38 papers have been published on subterranean Crustacea fauna of Poland (Table 3). Among all crustaceans (95 taxa) found in caves, wells and interstitial (inland) waters in Poland, only 24 taxa are stygobionts (nine Amphipoda; one Isopoda; four Copepoda; nine Ostracoda and one Bathynellid) (Table 3).

Based on the literature, the occurrences of crustaceans in subterranean waters of Poland were classified in Table 3 by habitat types (caves, wells and inland interstitial waters). The most diverse fauna was found in wells (59 taxa), then in interstitial waters (44) and in caves (31). Among Ostracoda, unfortunately for seven taxa there are no data available on habitat type and region where they were found (Table 3).

Table 3. List of Crustacean species recorded from subterranean aquatic habitats in Poland along with the data on ecology (subterranean habitat type) and geographical distribution (regions according to Catalogus Faunae Poloniae). Abbreviations of geographic names: Mts—mountains; Upl.—upland; Low.-lowland.

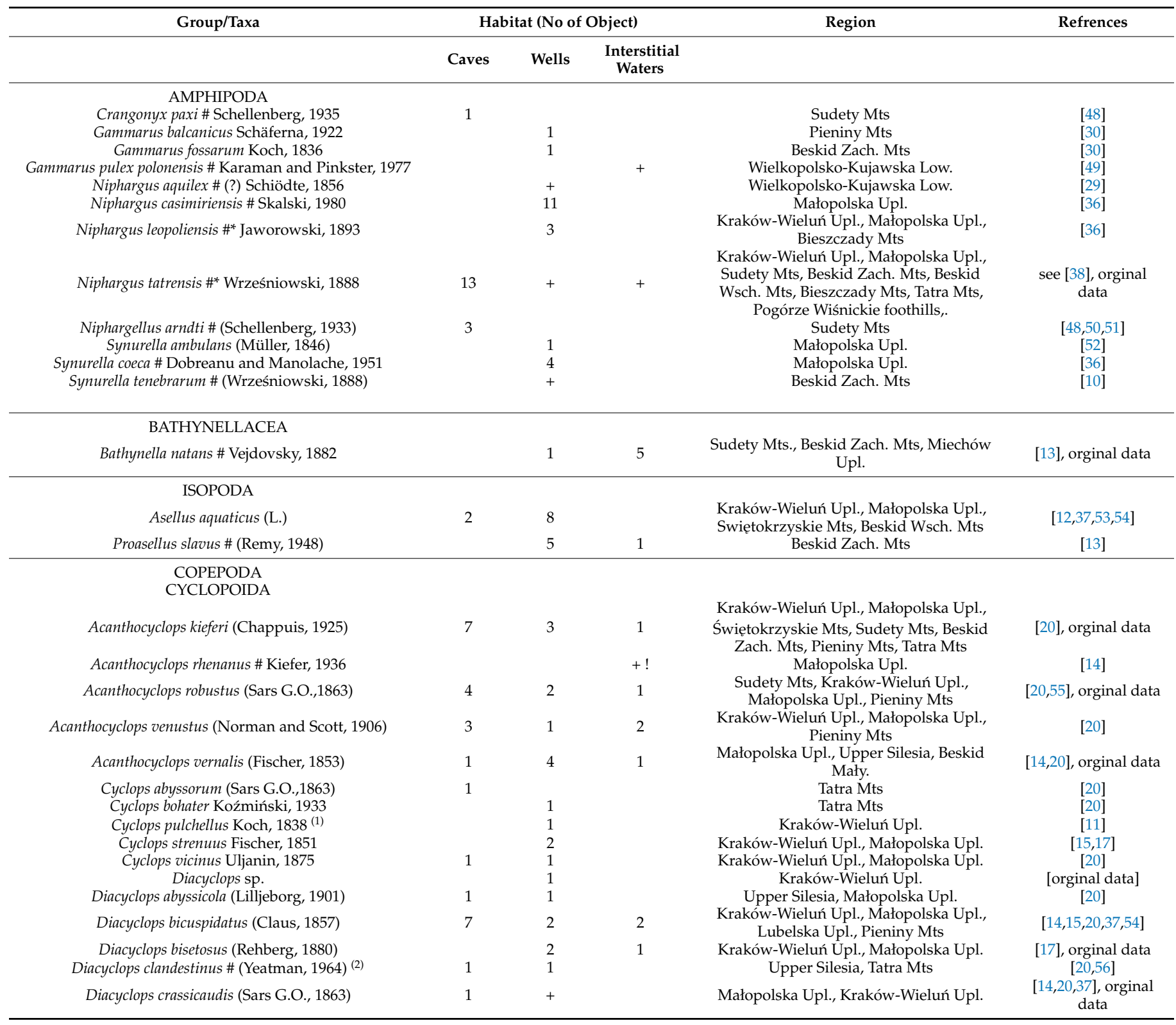


Table 3. Cont.

\begin{tabular}{|c|c|c|c|c|c|}
\hline \multirow[t]{2}{*}{ Group/Taxa } & \multicolumn{3}{|c|}{ Habitat (No of Object) } & \multirow[t]{2}{*}{ Region } & \multirow[t]{2}{*}{ Refrences } \\
\hline & Caves & Wells & $\begin{array}{l}\text { Interstitial } \\
\text { Waters }\end{array}$ & & \\
\hline Diacyclops crassicaudis brachycercus (Kiefer, 1927) & & 1 & & Małopolska Upl. & [orginal data] \\
\hline Diacyclops disjunctus (Thallwitz, 1927) ${ }^{(3)}$ & 2 & & & Sudety Mts., & [57] \\
\hline Diacyclops languidoides (Lilljeborg, 1901) & 5 & 1 & 1 & $\begin{array}{c}\text { Upper Silesia, Małopolska Upl., Beskid } \\
\text { Zach. Mts, Pieniny Mts }\end{array}$ & {$[15,20]$} \\
\hline Diacyclops languidus (Sars G.O., 1863) & & 1 & & Małopolska Upl. & [15] \\
\hline Diacyclops nanus (Sars G.O., 1863) & 1 & 1 & & Kraków-Wieluń Upl., Małopolska Upl. & {$[20]$} \\
\hline Eucyclops macruroides (Lilljeborg, 1901) & & 2 & 2 & Upper Silesia, Małopolska Upl. & {$[14,20]$} \\
\hline Eucyclops serrulatus (Fischer, 1851) & 2 & 6 & 3 & $\begin{array}{l}\text { Kraków-Wieluń Upl., Małopolska Upl., } \\
\text { Świetokrzyskie Mts, Pieniny Mts }\end{array}$ & {$[14,15,20]$} \\
\hline Eucyclops speratus (Lilljeborg, 1901) & & 1 & & Małopolska Upl. & [14] \\
\hline Graeteriella unisetigera \# (Graeter, 1908) & & 1 & & $\begin{array}{l}\text { Wielkopolsko-Kujawska Low. } \\
\text { Upper Silesia Kraków-Wieluń Upl }\end{array}$ & {$[16]$} \\
\hline Macrocyclops albidus (Jurine, 1820) & 1 & 1 & 3 & $\begin{array}{c}\text { Małopolska Upl., Lubelska Upl., Sudety } \\
\text { Mts }\end{array}$ & {$[14,17,20]$} \\
\hline Macrocyclops fuscus (Jurine, 1820) & & 1 & & Małopolska Upl. & [14] \\
\hline Megacyclops gigas (Claus,1857) & & & 1 & Pieniny Mts & [20] \\
\hline Megacyclops viridis (Jurine, 1820) & 4 & 2 & & $\begin{array}{c}\text { Kraków-Wieluń Upl., Małopolska Upl., } \\
\text { Sudety Mts, Beskid Mały }\end{array}$ & $\begin{array}{l}{[14,20,54,57]} \\
\text { orginal data }\end{array}$ \\
\hline Metacyclops sp. & 1 & & & Kraków-Wieluń Upl. & [55] \\
\hline Paracyclops affinis (Sars G.O., 1863) & 1 & 1 & & Kraków-Wieluń Upl., Małopolska Upl. & {$[20,37]$} \\
\hline Paracyclops fimbriatus (Fischer, 1853) & 1 & 4 & 1 & $\begin{array}{c}\text { Kraków-Wieluń Upl., Małopolska Upl., } \\
\text { Beskid Zach. Mts }\end{array}$ & {$[15,17,37]$} \\
\hline Paracyclops imminutus Kiefer 1929 & & 1 & & Beskid Mały & [orginal data] \\
\hline Paracyclops poppei (Rehberg, 1880) & 2 & 2 & & $\begin{array}{c}\text { Kraków-Wieluń, Małopolska Upls, } \\
\text { Sudetes, Beskid Zach. Mts }\end{array}$ & {$[20]$} \\
\hline $\begin{array}{l}\text { Thermocyclops crassus (Fischer, 1853) } \\
\text { Tropocyclops prasinus (Fischer, 1860) } \\
\text { CALANOIDA }\end{array}$ & 1 & 1 & & $\begin{array}{l}\text { Małopolska Upl. } \\
\text { Beskid Mały }\end{array}$ & $\stackrel{[20]}{\text { [orginal data] }}$ \\
\hline $\begin{array}{c}\text { Eudiaptomus graciloides (Lilljeborg, 1888) } \\
\text { HARPACTICOIDA }\end{array}$ & & 1 & & Małopolska Upl. & [15] \\
\hline Attheyella wierzejskii (Mrázek, 1893) & & 1 & 1 & Małopolska Upl., Sudety Mts & [20] \\
\hline Bryocamptus cuspidatus (Schmeil, 1893) & & 1 & & Małopolska Upl. & [58] \\
\hline Bryocamptus dacicus (Chappuis, 1923) & 2 & & & Sudety Mts & [57] \\
\hline Bryocamptus echinatus (Mrázek, 1893) & & 1 & & Małopolska Upl. & [58] \\
\hline Bryocamptus typhlops (Mrázek, 1893) & 1 & & & Sudety Mts & [57] \\
\hline Canthocamptus microstaphylinus (Wolf, 1909) & 1 & & 1 & Małopolska Upl., Lubelska Upl. & [19] \\
\hline Canthocamptus staphylinus (Jurine, 1820) & 1 & & 1 & Małopolska Upl., Lubelska Upl. & [19] \\
\hline Elaphoidella elaphoides \# (Chappuis, 1924) & & 4 & & Podlasie Low., Małopolska Upl. & {$[18,58]$, orginal data } \\
\hline Elaphoidella cf. elaphoides \# & & 1 & & Pogórze Wiśnickie foothills & [orginal data] \\
\hline Epactophanes richardi Mrázek, 1893 & 1 & & & Sudety Mts., & [57] \\
\hline Nitokra hibernica hyalina Jakubisiak, 1929 & & & & Wielkopolsko-Kujawska Low. & {$[59]$} \\
\hline Mesochra sp. (Schmeil, 1894) & & 1 & + & Małopolska Upl. & [orginal data] \\
\hline \multicolumn{6}{|l|}{ OSTRACODA } \\
\hline Bradleystrandesia reticulata (Zaddach, 1844) ${ }^{(4)}$ & $\begin{array}{l}\text { no details } \\
\text { on } \\
\text { habitats }\end{array}$ & & & no details on sites & [24] \\
\hline Candona candida (O.F. Müller, 1776) & & + & + & $\begin{array}{c}\text { Kraków-Wieluń Upl., Sudety Mts, Beskid } \\
\text { Zach. Mts, Bieszczady Mts, Małopolska } \\
\text { Upl. Wielkopol.-Kujawska Low, Lublin } \\
\text { Upl.. }\end{array}$ & {$[24,26,36,60,61]$} \\
\hline Cavernocypris subterranea (Wolf, 1919) & &,+ 2 & + & $\begin{array}{c}\text { Sudety Mts, Beskid Zach. Mts, Bieszczady } \\
\text { Mts, Kraków-Wieluń Upl., Małopolska } \\
\text { Upl. }\end{array}$ & $\begin{array}{l}{[24,26,60,61]} \\
\text { orginal data }\end{array}$ \\
\hline Cryptocandona sp. & & 1 & & Pogórze Wiśnickie foothills & [orginal data] \\
\hline Cryptocandona matris \# (Sywula, 1976) & & + & & $\begin{array}{l}\text { Małopolska Upl., Beskid Zach. Mts, Beskid } \\
\text { Wsch. Mts, Bieszczady Mts, Pieniny Mts, } \\
\text { Lublin Upl., Pogórze Wiśnickie foothills }\end{array}$ & $\begin{array}{l}{[23-25,27,28,36]} \\
\quad \text { orginal data }\end{array}$ \\
\hline Cryptocandona reducta $(\mathrm{Alm}, 1914)$ & & + & + & Sudety Mts, other sites & {$[24,27,60,61]$} \\
\hline Cryptocandona vavrai Kaufmann, 1900 & & + & + & Sudety Mts, Beskid Zach., Tatra Mts & {$[24,27,60,61]$} \\
\hline Cyclocypris ovum (Jurine, 1820) & $\begin{array}{l}\text { no details } \\
\text { on } \\
\text { habitats }\end{array}$ & 1 & & $\begin{array}{c}\text { no details on sites, Pogórze Wiśnickie } \\
\text { foothills }\end{array}$ & [24], orginal data \\
\hline Cyclocypris serena (Koch, 1838) & $\begin{array}{l}\text { no details } \\
\text { on } \\
\text { habitats }\end{array}$ & & & no details on sites & [24] \\
\hline Cyclocypris cf. serena (Koch, 1838) & & 1 & & Kraków-Wieluń Upl. & [orginal data] \\
\hline Cypria ophtalmica (Jurine, 1820) & & + & + & $\begin{array}{c}\text { Sudety Mts, Beskid Zach. Mts., Pogórze } \\
\text { Wiśnickie foothills }\end{array}$ & [24], orginal data \\
\hline Cypria reptans Bronstein, 1928 & & & + & no details on sites & [24] \\
\hline Darwinula stevensoni (Brady and Robertson, 1870) & & & + & no details on sites & {$[24]$} \\
\hline Eucypris pigra (Fischer, 1851) & & & + & no details on sites & {$[24]$} \\
\hline Fabaeformiscandona breuili \#* (Paris, 1920) ${ }^{(5)}$ & & + & + & $\begin{array}{c}\text { Sudety Mts, Bieszczady Mts, Pieniny Mts, } \\
\text { Beskid Zach. Mts, Wielkopolsko-Kujawska } \\
\text { Low. }\end{array}$ & {$[22,24,25,28]$} \\
\hline Fabaeformiscandona brevicornis (Klie, 1925) ${ }^{(6)}$ & & 1 & + & no details on sites, Beskid Mały & [24], orginal data \\
\hline Fabaeformiscandona latens \#* (Klie, 1940) & & & + & Sudety Mts, Beskid Zach. Mts. & {$[22,24]$} \\
\hline
\end{tabular}


Table 3. Cont.

\begin{tabular}{|c|c|c|c|c|c|}
\hline \multirow[t]{2}{*}{ Group/Taxa } & \multicolumn{3}{|c|}{ Habitat (No of Object) } & \multirow[t]{2}{*}{ Region } & \multirow[t]{2}{*}{ Refrences } \\
\hline & Caves & Wells & $\begin{array}{l}\text { Interstitial } \\
\text { Waters }\end{array}$ & & \\
\hline Fabaeformiscandona wegelini \#* (Petkovski, 1962) & & + & + & $\begin{array}{c}\text { Sudety Mts, Beskid Zach. Mts, Małopolska } \\
\text { Upl., Wielkopolsko-Kujawska Low., Lublin } \\
\text { Upl.. }\end{array}$ & {$[22,24,36]$} \\
\hline Herpetocypris sp. & $\begin{array}{l}\text { no details } \\
\text { on } \\
\text { habitats }\end{array}$ & & & no details on sites & [24] \\
\hline Ilyocypris bradyi Sars, 1890 & & & + & no details on sites & [24] \\
\hline Limnocythere inopinata (Baird, 1843) & & & + & no details on sites & [24] \\
\hline Mixtacandona sp. \# & & + & & Małopolska Upl. & {$[24,36]$} \\
\hline Nannocandona faba Ekman, 1914 & & + & + & Sudety Mts & [24] \\
\hline Nannocandona stygia \# Sywula, 1976 & & + & + & Sudety Mts, Beskid Zach. Mts. & {$[23,24]$} \\
\hline Neglecandona lindneri (Petkovski, 1969) & $\begin{array}{l}\text { no details } \\
\text { on } \\
\text { habitats }\end{array}$ & & & no details on sites & [24] \\
\hline Neglecandona neglecta (Sars, 1887) & & & + & no details on sites & [24] \\
\hline Notodromas monacha (O.F. Müller, 1776) & $\begin{array}{l}\text { no details } \\
\text { on } \\
\text { habitats }\end{array}$ & & & no details on sites & [24] \\
\hline Physocypria kraepelini G.W. Müller, 1903) ${ }^{(7)}$ & & & + & no details on sites & [24] \\
\hline Potamocypris fulva (pallida) (Brady, 1868) & & + & + & Sudety Mts, Beskid Zach, Bieszczady Mts & [24] \\
\hline Potamocypris cf. pallida (Alm, 1914) & & 1 & & Małopolska Upl. & [orginal data] \\
\hline Potamocypris zschokkei (Kaufman, 1900) ${ }^{(8)}$ & & & + & $\begin{array}{c}\text { no details on sites, except Kraków-Wieluń } \\
\text { Upl. }\end{array}$ & {$[24,26]$} \\
\hline Pseudocandona albicans (Brady, 1864) ${ }^{(9)}$ & & + & + & $\begin{array}{l}\text { Małopolska Upl., Kraków-Wieluń Upl., } \\
\text { Sudety Mts, Beskid Zach. Mts }\end{array}$ & {$[21,22,24-26,36]$} \\
\hline Pseudocandona compressa (Koch, 1838) & & + & & no details on sites & [24] \\
\hline Pseudocandona mira \# (Sywula, 1976) & & + & & Beskid Zach. Mts & [23] \\
\hline Pseudocandona pratensis (Hartwig, 1901) & & + & & Sudety Mts and other sites & {$[24,60]$} \\
\hline Pseudocandona sarsi (Hartwig,1899) & + & + & + & $\begin{array}{l}\text { Małopolska Upl., Kraków-Wieluń Upl., } \\
\text { Sudety Mts, Beskid Zach. Mts }\end{array}$ & {$[24,37,54,60]$} \\
\hline Pseudocandona semicognita (Schafer, 1934) & $\begin{array}{l}\text { no details } \\
\text { on } \\
\text { habitats }\end{array}$ & & & no details on sites & [24] \\
\hline Pseudocandona triquetroides (Sywula, 1974) & & & + & Sudety Mts, Beskid Zach. Mts & [24] \\
\hline Typhlocypris eremita \#*(Vejdovsky, 1882) & & + & & $\begin{array}{c}\text { Sudety Mts, Małopolska Upl., } \\
\text { Wielkopolsko-Kujawska Low., Beskid } \\
\text { Zach. Mts, Lublin Upl.. }\end{array}$ & $\begin{array}{c}{[24,36],} \\
\text { Sywula-pers. notes }\end{array}$ \\
\hline Typhlocypris cf. eremita (Vejdovsky, 1882) & & 2 & & Kraków-Wieluń Upl., Małopolska Upl. & [orginal data] \\
\hline Typhlocypris szoecsi \# (Farkas, 1958) & & & & $\begin{array}{c}\text { Małopolska Upl., Beskid Zach. Mts, Lublin } \\
\text { Upl.. }\end{array}$ & {$[24,36]$} \\
\hline
\end{tabular}

+—presence taxon confirmation. \#—stygobiontic species. * - for stygobionts only: found also in surface waters. !-found in peat bog forest Reservoirs 1, 2, and 3- "original" names of species used in cited papers. ${ }^{(1)}$-C. pulchellus Koch, 1838 is currently regarded as nomen dubium; in the past some carcinologists used this name as a senior synonym of Diacyclops bicuspidatus (Claus, 1857). (2)—as Diacyclops clandestinus (Kiefer, 1926) in [56]. (3) —as Diacyclops languidus disjunctus (Thallwitz, 1927) in [57]. ${ }^{(4)}$ as Cypricercus affinis (Fischer, 1851) in [24]. ${ }^{(5)}$-as Candona hertzogi beskidana (Sywula 1974) in $[22,24,25,28] .{ }^{(6)}$ —as Candona limnocrenica (Sywula 1971) in [24]. (7) —as Physocypria fadeewi (Dubovsky 1926) in [24]. ${ }^{(8)}$ —as Potamocypris foxi (Sywula 1972) in [24,26]; P. wolfi (Brehm 1920) in [24]. ${ }^{(9)}$ —as Candona parallela (G.W. Muller) 1900 in $[21,22,24-26,36]$.

\section{Discussion}

The subterranean fauna dwells in underground waters such as caves, interstitial waters, wells, as well as other man-made subterranean habitats such as adits, shafts or mines. It also occurs in springs [3].

Although several samples did not yield any crustaceans, the results suggest that the sampling effort was adequate to represent crustacean communities in the wells. The recorded 23 (sub-)species of Crustacea in the wells amounted to $73.5 \%$ of the estimated species richness (Figure 3), a value within the range (50-75\%) which Heck et al. [62] consider an adequate approximation.

The values of physical and chemical parameters of water in wells located in different bedrocks (including flysch rocks of the Carpathians, Jurassic limestone sedimentary rocks and Cretaceous marls) differed. The mean values from wells located in Jurassic limestone (SZ, Wi) and cretaceous marls (Pr) bedrock were usually similar, but wells located on flysch bedrock ( $\mathrm{Ja}, \mathrm{Ka})$ had mostly lower means that may be related to lower mineralization. It should be emphasized that the water in the studied wells was neutral to slightly alkaline. The increased levels of nitrates, chlorides and sulfates in some wells indicate a significant human impact on the quality of the groundwater. Similar results concerning quality of groundwater were observed earlier in two regions, in the Kraków-Częstochowa upland [63,64] 
and in the Wiśnickie foothill $[65,66]$. In regions where polluted wells predominate, the species richness and abundance of the crustecean fauna is much smaller [3].

The copepod stygobiont Elaphoidella elaphoides, previously was found only in wells in the villages of Ogrodniczki and Ciasne in Poland (Uplands of the Podlaskie Plain) $[18,58]$. In Europe, this stygobiont is widely distributed in underground waters, including caves, hyporheic and phreatic waters and often occurs in epigean waters as well [18].

Two stygobiontic ostracod species, Cryptocandona matris in Kawec and Typhlocypris cf. eremita occurred in Szklary and Prandocin wells. The former species was originally described by Sywula [23] from a well at Cisna village in the Bieszczady Mts. and further recorded in wells and interstitial habitats of the Lublin Upland and Carpathian Mountains (in Poland) (Table 3) and in north-eastern Romania [27]. Typhlocypris eremita is the type and the most-widespread species of the genus, occurring in groundwaters of Central and South-Eastern Europe [46], mainly as all-female (parthenogenetic) populations. As the male genital morphology offers better characteristics than that of female on which to define the species in the genus Typhlocypris, it is not unlikely that some of recorded populations could represent different species, as documented by Iepure et al. [67]. Thus, a re-examination of records identified as Typhlocypris eremita is required to better understand the extent of variation of this and closely related species. In Poland, T. eremita is known mostly from an area south of the maximum limit of the Vistulian glaciation, with the most significant exception of two surface-water sites in the Vistula fens in northern Poland (Table 3). These are the northern-most localities of this species, which were most probably reached by this species via the alluvial groundwaters of the Vistula River [68,69].

The amphipod stygobiont Niphargus tatrensis previously was reported from the wells situated in the Kraków-Czestochowa upland [3], and is common in southern Poland [38]. It was found in the Prandocin well (Pogórze Wiśnickie foothill) and is the first record of this species from this region. Other species of crustaceans found in the studied wells were nonobligate groundwater inhabitants occurring mainly in surface inland waters. Considering ostracods, all the remaining non-stygobite species collected during this study, have been already recorded in groundwaters of Poland [24] (Table 3). Two of these, Cavernocypris subterranea and Fabaeformiscandona brevicornis may qualify as stygophiles or crenobionts, as they inhabit both groundwaters and surface waters associated with springs [24,44].

Statistical analyzes showed that the species richness and abundance of crustacean fauna in the studied wells depended especially on the bedrock in which the wells are located, and not on the measured chemical and physical parameters of water. For Ostracoda growth and survival may be greatly affected by the solute composition and concentration of major ions in water. In waters depleted in calcium and magnesium ostracod shell calcification at moulting may be disturbed, resulting in development of not fully calcified, soft carapaces [70]. It seems that wells localized in the geological formation of the Cretaceous marls and characterized by the highest average calcium concentration in water, proved to represent the habitat successful for ostracoda populations. Ostracods in the Prandocin wells had indeed the highest abundances and species richness. The low abundance and number of copepods species in Jurassic wells could be influenced by pollution of some of them (especially in $\mathrm{Wi}$ ). Results based on various groups of benthic fauna (excluding microcrustaceans) studied in the wells located in the flysch and limestone regions showed that the parameters of water chemistry related to the pollution and depth of the studied wells influenced the diversity, composition and abundance of the fauna [3]. However, it was possible that other constrains, including water properties (including that not measured during this survey) and/or sediment type and some biological factors might also play important role in determining the demonstrated differences in crustacean alpha diversity and abundances between subterranean waters of the studied geological formations. Groundwater invertebrates in Poland have been studied from different perspectives, including a focus on regions, habitats and finding stygobiontic species. The south of Poland is the best studied region, the north is the weakest. In Poland, up to now among the total species number of crustaceans (95 taxa) found in groundwater (caves, wells and interstitial 
waters), only 24 obligate stygobiont species were recorded (nine species of amphipods, one bathynellacean, one isopod, four copepods and nine ostracods) (Table 3). The most diverse groups are copepods and ostracods. Most stygobionts have a narrow range, so the risk of species extinction is particularly high in the face of the increase in multiple anthropogenic pressures [71,72]. Our study showed that the literature concerning Polish crustacean fauna from subterranean waters (including stygobiotnic species) is still limited and thus provides an opportunity for further study. Especially the crustaceans fauna in interstitial water has been weakly studied, resulting in a small number of species known from this habitat. Knowledge on the diversity of faunal communities that live in wells can be used to monitor, protect, and manage the environment and can be useful for public health by indicating local water pollution.

Author Contributions: Conceptualization, A.P. and E.D.; investigation, E.D. and J.G.; laboratory analyses, A.P., M.K. and T.N.; software, M.K. and T.N.; writing-original draft preparation, A.P., M.K., T.N.; writing - review and editing, A.P., T.N., J.G. and E.D.; visualization, A.P. and M.K.; supervision, A.P. All authors have read and agreed to the published version of the manuscript.

Funding: This research was funded by subvention of Institute of Nature Conservation, Polish Academy of Sciences (A.P., E.D., J.G.) and partly by the internal grant of the University of Gdansk No. 531-D090-D818-21 attributed to T.N.

Institutional Review Board Statement: Not applicable.

Informed Consent Statement: Not applicable.

Data Availability Statement: The original contributions presented in the study are included in the article. Further inquiries can be directed to the corresponding author.

Acknowledgments: We would like to thank Hanna Kuciel and Andrzej Kalemba for their help with drawing works. We extend our thanks to Henri Dumont for the assistance in language editing.

Conflicts of Interest: The authors declare no conflict of interest.

\section{References}

1. Gibert, J.; Culver, D.C. Assessing and conserving groundwater biodiversity: An introduction. Freshw. Biol. 2009, 54, 639-648. [CrossRef]

2. Sket, B. High biodiversity in hypogean waters and its endangerment-the situation in Slovenia, the Dinaric karst, and Europe. Crustaceana 1999, 72, 767-779. [CrossRef]

3. Dumnicka, E.; Galas, J.; Krodkiewska, M. Patterns of benthic fauna distribution in wells: The role of anthropogenic impact and geology. Vadose Zone J. 2017, 16, 1-9. [CrossRef]

4. Kayo, R.T.; Mormonier, P.; Togouet, S.H.Z.; Nola, M.; Piscart, C. An annotated checklist of freshwater Stygobiontic crustaceans of Africa and Madagascar. Crustaceana 1912, 85, 1613-1631.

5. ̌eháčková, V. Well-water organisms of Prague. Rozpr. Cesk. Akad. Ved. 1953, 63, 1-35.

6. Kulhavý, V. Beitrag zur Kenntnis der Crustaceenfauna tschechoslowakischer Grundgewässer. Vestn. Cesk. Spol. Zool. 1960, 24, 287-306.

7. Terek, J.; Brázda, J. Fauna of wells outside settlements on Východoslovenská nižina (Czechoslovakia). Biologia 1986, 41, 971-979.

8. Hahn, H.J.; Fuchs, A. Distribution patterns of groundwater communities cross aquifer types in south-western Germany. Freshw. Biol. 2009, 54, 848-860. [CrossRef]

9. Arnscheidt, J.; Hahn, H.J.; Fuchs, A. Aquatic subterranean Crustacea in Ireland: Results and new records from a pilot study. Cave Karst Sci. 2008, 35, 53-58.

10. Wrześniowski, A. O trzech kiełżach podziemnych. Pamiętnik Fizyogr. 1888, 8, 221-330.

11. Jaworowski, A. Fauna studzienna miast Krakowa i Lwowa. Spraw. Kom. Fizyograf. AU W Krakowie 1893, $28,29-48$.

12. Skalski, A.W. Ośliczka Asellus aquaticus (L.) (Isopoda) w wodach podziemnych południowej Polski. V Rocz. Muz. Okręgowego W Częstochowie Przyr. 1981, 2, 85-88.

13. Sywula, T. Bathynella natans Vejdovsky, 1882 i Proasellus slavus (Remy, 1948), nowe dla Polski podziemne skorupiaki. Prz. Zool. 1989, 33, 77-82.

14. Tabacki, A. Widłonogi (Copepoda) rzeki Grabi (część faunistyczna). Zesz. Nauk. Uniw. Łódź, seria II, Nauki Mat.-Przyr. 1971, 44, 31-52.

15. Tabacki, A. Widłonogi (Copepoda) wód studziennych okolic Sulejowa koło Łodzi. Acta Univ. Lodz. Zeszyty Naukowe UŁ. Nauki Mat-Przyr. Ser. II 1980, 33, 123-128. 
16. Wróblewski, A.; Sywula, T. Copepoda-Widłonogi. In Checklist of animals of Poland; Razowski, J., Ed.; ISEZ PAN: Kraków, Poland, 1997; pp. 158-164.

17. Czaja, M. Widłonogi Copepoda Cyclopoida Ojcowskiego Parku Narodowego. Master's Thesis, University of Gdansk, Gdansk, Poland, 1999.

18. Karpowicz, M. New data to the knowledge on the Harpacticoida (Crustacea, Copepoda) fauna in Poland. Fragm. Faun. 2016, 59, 87-98.

19. Kur, J. Zmienność Populacyjna Widłonogów Copepoda w Wodach Podziemnych Południowej Polski. Praca Doktorska; IOP Publishing: Kraków, Poland, 2012.

20. Kur, J.; Mioduchowska, M.; Kilikowska, A. Distribution of cyclopoid copepods in different subterranean habitats (southern Poland). Oceanol. Hydrobiol. Stud. 2020, 49, 255-266. [CrossRef]

21. Sywula, T. The ostracods (Ostracoda) of the River Raba and of certain aquatic environments connected with it. Acta Hydrobiol. 1974, 16, 255-277.

22. Sywula, T. Notes on Ostracoda. XIV. Interesting species from the basin of the River Raba (South Poland). Ann. Zool. 1974, 32, 59-73.

23. Sywula, T. New species of Ostracoda (Crustacea) from subterranean waters of Poland. Bull Acad. Pol. Sci. Ser. Sci. Biol. Cl II 1976, 24, 271-278.

24. Sywula, T. Małżoraczki (Ostracoda) wód podziemnych Polski. V Rocznik Muzeum Okręgowego W Częstochowie Przyroda 1981, 2, 89-96.

25. Sywula, T. Skorupiaki (Crustacea) Bieszczadów (z wyjątkiem Malacostraca). Monogr. Bieszcz. 2000, 7, 49-54.

26. Skalski, A.W. Flora i fauna jaskiń Wyżyny Krakowsko-Częstochowskiej. I Sympozjum Jurajskie Człowiek i środowisko naturalne Wyżyny Krakowsko-Wieluńskiej. Dąbrowa Górnicza 5-6 V; Dabrowa Górnicza: Silesia, Poland, 1992; Volume 1993, pp. 145-187.

27. Baltanás, A.; Namiotko, T.; Danielopol, D.L. Biogeography and disparity within the genus Cryptocandona (Crustacea, Ostracoda). Vie Et Milieu Life Environ. 2000, 50, 297-310.

28. Sywula, T.; Jędryczkowski, W.B. Skorupiaki (Crustacea). In Flora i Fauna Pienin; Razowski, J., Ed.; Monografie Pienińskie: Krościenko, Poland, 2000; pp. 107-110.

29. Von Haeckel, K. Niphargus aquilex im Odergebiet. Zool. Anz. 1907, 32, 430-431.

30. Micherdziński, W. Kiełże rodzaju Gammarus FABRICIUS (Amphipoda) w wodach Polski. Acta Zool. Crac. 1959, 4, $527-637$.

31. Skalski, A.W. Niphargus casimiriensis sp.n., a new species of hypogeous amphipod from Poland (Crustacea, Niphargidae). Acta Hydrobiol. 1980, 22, 217-227.

32. Skalski, A.W. Niphargus leopoliensis Jaworowski, 1983 (Amphipoda) in Poland. Przeglad Zool. 1980, 24, 97-101.

33. Skalski, A.W. The hypogeous gammarids in Poland (Crustacea, Amphipoda, Gammaridae). Acta Hydrobiol. $1970,12,431-437$.

34. Skalski, A.W. Distribution des Amphipodes souterrains en Pologne, avec notes sur la variabilite du Niphargus tatrensis Wrześniowski. In Proceedings of the Actes du Ier Colloque International sur le genre Niphargus, Verona, Italy, 15-19 April 1969.

35. Skalski, A.W. Podziemne obunogi (Amphipoda) Polski. V Rocznik Muzeum Okregowego W Częstochowie. Przyroda 1981, 2, 61-83.

36. Skalski, A.W. Groundwater fauna of the Małopolska Gap of the Vistula. Pol. Arch. Hydrobiol. 1982, $29,387-404$.

37. Konopacka, A.; Jażdżewski, K. Groundwater crustaceans of the wells in the Grabia river system (Central Poland). In Proceedings of the 2nd European Crustacean Conference, Liege, Belgium, 2-6 September 1996.

38. Dumnicka, E.; Galas, J. An overview of stygobiontic invertebrates of Poland based on published data. Subterr. Biol. 2017, 23, 1-18. [CrossRef]

39. Karpowicz, M.; Pociecha, A.; Hołyńska, M.; Dumnicka, E.; Galas, J. Groundwater copepods from Carpathian flysch in south Poland. In Proceedings of the III Krajowa Konferencja Zooplanktonowa, Szczecinek, Poland, 20-22 June 2018.

40. Janetzky, W.; Enderle, R.; Noodt, W. Bd. 8/4, 2: Crustacea: Copepoda: Gelyelloida und Harpacticoida; Fischer: Stuttgart, Germany, 1996.

41. Błędzki, L.A.; Rybak, J.I. Freshwater Crustacean Zooplankton of Europe: Cladocera E Copepoda (Calanoida, Cyclopoida). Key to Species Identification, with Notes on Ecology, Distribution, Methods and Introduction to Data Analysis; Springer: Berlin/Heidelberg, Germany, 2016.

42. Pesce, G.L.; Galassi, D.P. New or rare species of Diacyclops Kiefer, 1927 (Copepoda, Cyclopoida) from different groundwater habitats in Italy. Hydrobiologia 1987, 148, 103-114. [CrossRef]

43. Sywula, T. Małżoraczki Ostracoda; PWN: Warszawa-Poznań, Poland, 1974.

44. Meisch, C. Freshwater Ostracoda of Western and Central Europe; Spektrum Akademischer: Heidelberg, Germany, 2000.

45. Namiotko, T.; Danielopol, D.L.; Meisch, C.; Gross, M.; Mori, N. Redefinition of the genus Typhlocypris Vejdovský, 1882 (Ostracoda, Candonidae). Crustaceana 2014, 87, 952-984. [CrossRef] [PubMed]

46. Namiotko, T.; Danielopol, D.L.; Baltanás, A. Soft body morphology, dissection and slide-preparation of Ostracoda: A primer. Joannea Geologie Und Paläontologie 2011, 11, 327-343.

47. Clarke, K.R.; Gorley, R.N. PRIMER v7: User Manual/Tutorial; PRIMER-E Ltd.: Plymouth, UK, 2015.

48. Schellenberg, A. Die Höhlenfauna des Glazer Schneeberges. 2. Höchlenamphipoden des Glatzer Schneeberges. Beitrage zur Biologie des Glatzer Schneeberges 1935, 1, 72-75.

49. Karaman, G.S.; Pinkster, S. Freshwater Gammarus species from Europe, North Africa and adjacent regions of Asia (CrustaceaAmphipoda). Part I. Gammarus pulex-group and related species. Bijdragen Tot De Dierkunde 1977, 47, 1-97. [CrossRef] 
50. Hajduk, Z.; Ogorzałek, A. Niphargellus arndti (Schellenberg, 1933) z jaskini Kontaktowej koło Kletna. Acta Univ. Wratislav. 3. Stud. Geograf. 1978, 24, 155-157.

51. Lorenc, M.W.; Chlebicki, A.; Szumska, E. Mieszkańcy dawnych wyrobisk kopalni złota w Złotym Stoku. In Proceedings of the Materiały 37 Sympozjum Speleologicznego, Wojcieszów, Poland, 24-26 October 2003.

52. Konopacka, A.; Sobocińska, V. Uwagi na temat występowania skorupiaka Synurella ambulans (Müll.) (Amphipoda, Crangonyctidae) w Polsce. Przegl. Zool. 1992, 36, 123-131.

53. Dumnicka, E.; Wojtan, K. Faune aquatique de la grotte Chelosiowa Jama (Montagnes Świętokrzyskie, Pologne Centrale). Mém. Biospéol. 1994, 21, 53-56.

54. Dumnicka, E.; Galas, J.; Karlikowska, J.; Sznober, N. Temporary co-existence of aquatic and terrestrial invertebrates in shallow periodically flooded and frozen cave. Biologia 2015, 70, 1201-1209. [CrossRef]

55. Dumnicka, E.; Wojtan, K. Differences between cave water ecological systems in the Kraków—Częstochowa Upland. Stygologia 1990, 5, 241-247.

56. Chodorowska, W.; Chodorowski, A. Ugrupowania fauny wodnej w jaskiniach tatrzańskich. Speleologia II 1960, 1, 57-62.

57. Stammer, H.J. Die Höhlenfauna des Glazer Schneeberges. 8. Die Wasserfauna der Schneeberghöhlen. Beitrage Zur Biologie Des Glatzer Schneeberges 1936, 2, 199-214.

58. Konopacka, A. Studnie Okolic Rzeki Grabi; University of Lodz: Lodz, Poland, 1993; Unpublished work.

59. Jakubisiak, S. Materiały do fauny skorupiaków widłonogich (Copepoda) z rodziny Harpacticidae w Poznańskiem i na Pomorzu. Spraw. Komisji Fizjograf. PAU 1931, 65, 31-65.

60. Klie, W. Candona latens, ein neuer Muschelkrebs aus dem Grundwasser von Mittelfranken. Zool. Anz. 1940, 131, 101-104.

61. Klie, W. Zur Kenntnis der Ostracodenarten Candona eremita (Vejdovsky) und Candona reducta Alm. Mitteilungen über Höhlen Und Karstforschung 1940, 1, 24-29.

62. Heck, K.L.; van Belle, G.; Simberloff, D. Explicit calculation of the rarefaction curves diversity measurement and the determination of sufficient sample size. Ecology 1975, 56, 1459-1461. [CrossRef]

63. Różkowski, J. Transformations in chemical composition of karst water in the southern part of the Cracow upland (Rudawa and Prądnik drainage areas). Kras Speleol. 1996, 1, 1-106.

64. Różkowski, J. Groundwaters of Carbonate Formations in the Southern Part of Jura Krakowsko-Częstochowska and Problems with Their Protection; University of Silesia: Katowice, Poland, 2006.

65. Ellis, J.; Dottridge, J. Nitrate contamination of shallow groundwater in the Carpathian foothills (southern Poland). Prace Geograf. 1998, 103, 147-157.

66. Żelazny, M. Biogenic Compounds in Precipitation Water, Streamwater and Groundwater in Catchments with Different Land Uses in the Wiśnickie Foothills, Southern Poland. Inst. of Geogr. and Spatial Manage; Jagiellonian University: Kraków, Poland, 2005.

67. Iepure, S.; Namiotko, T.; Danielopol, D.L. Evolutionary aspects within the species group Pseudocandona eremita (Vejdovský) (Ostracoda, Candonidae). Hydrobiologia 2007, 585, 159-180. [CrossRef]

68. Namiotko, T. Freshwater Ostracoda (Crustacea) of Żuławy Wiślane (Vistula Fen Country, Northern Poland). Acta Zool. Crac. 1990, 33, 459-484.

69. Namiotko, T.; Sywula, T. Zgrupowanie skorupiaków (Crustacea) rowów melioracyjnych koło Szymankowa na Żuławach Wiślanych. Zesz. Nauk. Uniw. Gdański Biologia 1993, 10, 159-162.

70. Mesquita-Joanes, F.; Viehberg, F.; Smith, A.J. The biology and ecology of ostracods. In Ostracoda as Proxies for Quaternary Climate Change. Developments in Quaternary Science Series; Horne, D.J., Holmes, J.A., Rodriguez-Lazaro, J., Viehberg, F.A., Eds.; Elsevier Science Publishing: Amsterdam, The Netherlands, 2012; Volume 17, pp. 15-35.

71. Malard, F.; Plenet, S.; Gibert, J. The use of invertebrates in ground water monitoring: A rising research field. Groundw. Monit. Remediat. 1996, 16, 103-113. [CrossRef]

72. Danielopol, D.L.; Griebler, C.; Gunatilaka, A.; Notenboom, J. Present state and future prospects for groundwater ecosystems. Environ. Conserv. 2003, 30, 104-130. [CrossRef] 